\title{
Design of Special Underwater Measurement and Control System Based
}

\section{on Distributed Management}

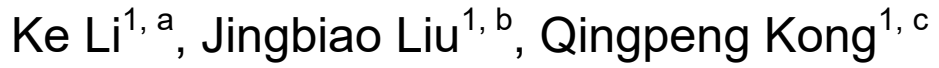 \\ ${ }^{1}$ College of Electronic \& Information, Hangzhou Dianzi University, Hangzhou, Zhejiang Province, \\ 310018, China \\ bemail: ab@hdu.edu.cn
}

Keywords: distributed management; measurement and control; extendibility; self-check

\begin{abstract}
Aiming at the rising demand for functional diversity and stability of underwater sampling equipment, a special underwater measurement and control system based on distributed management is designed in this paper. The system takes STM32F407 as MCU, dividing the whole function into different functional modules that work independently under unified management. Equipped with isolated CAN transceiver, each module connects to CAN bus in the method of board access so as to realize the centralized-distributed structure which takes the main control board as the management core. The system can be extended conveniently owing to its good access capability and extendibility. Besides, the system's self-check ability can help the system monitor the state of power supply and external control. Tests show that the system can realize efficient and stable operation and achieve all functions successfully.
\end{abstract}

\section{Introduction}

Underwater sampling equipment is the necessary equipment for marine geologic and environmental investigation [1]. Therefore, we attach great importance to its present situation and development. The update and improvement of the equipment may enhance the efficiency and accuracy of underwater exploration, getting higher economic and social benefits [2]. The new equipment puts forward higher requirements for the stability and the diversity of function of the measurement and control system.

A traditional analog instrument control system has a slice of disadvantages: all nodes work in the bottom and they are controlled by low-accuracy analog signals, which make the structure of system quite dispersed and vulnerable to interface. Overcoming the defects of an analog instrument system, a centralized enclosed digital measurement and control system can make judgment and control the system according to system's global state so as to accomplish the unified scheduling and arrangement. However, enough processing ability is indispensable for this system's core processor. If tasks turn to be more onerous, the efficiency and stability of system may rapidly fall off. What's more, lack of fault tolerance and extendibility will make the system hard to adapt tasks which are more complex and flexible [3] [4].

In this paper, an underwater measurement and control system based on distributed management structure which overcomes both defects of the above two is designed. Each independent functional processing module which is applied to its corresponding job sites connects to CAN bus network under the unified management of the senior main control module so that these modules cooperate with each other, establishing a complete centralized-distributed comprehensive system. The extendibility of such a distributed structure can help the system extend its function conveniently. Besides, self-check capability is fortified in this system which greatly reduces the dependency on 
artificial detection and promotes system's security and reliability.

\section{System design}

As is shown in figure.1, the system is composed of two parts: the measurement and control system under water and the monitoring system on the deck. Board access model is applied in the underwater system in which the main control board is responsible for the operation management to implement the data interaction and each functional board is responsible for implementing its own function. Functional boards consist of power management board, relay control board, acquisition boards and optical transceiver module. The monitoring computer combined with the optical transceiver device makes up the monitoring system on the deck. The communication method between these two parts is optical fiber communication.

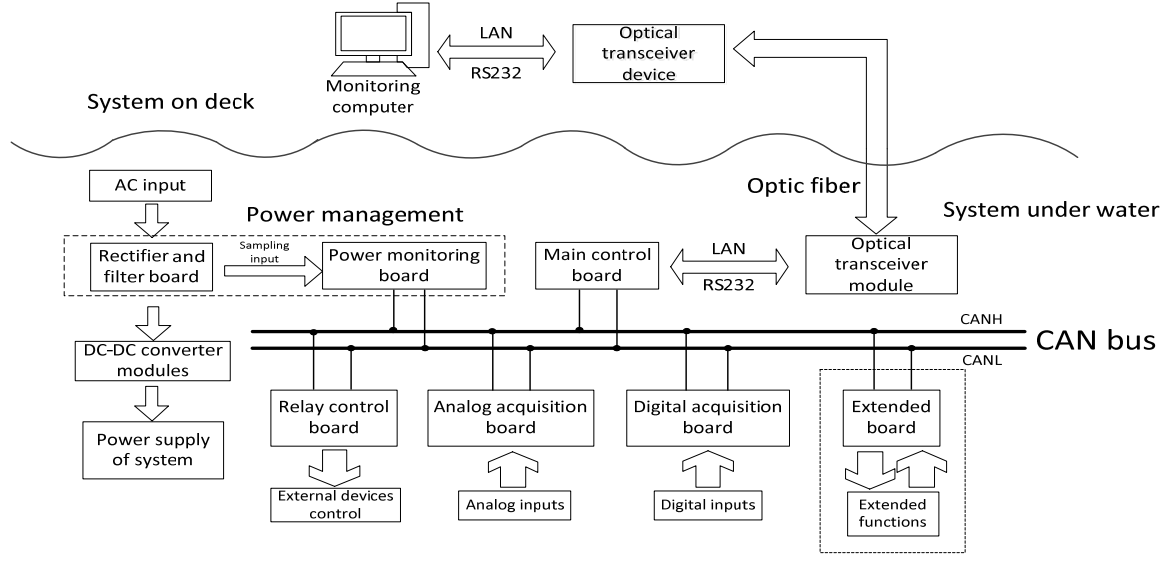

Figure.1. Overall system diagram

According to the design principle of this system, on the one hand, acquisition boards are in charge of data collection. The main control board receives data which comes from acquisition boards and sends to the monitoring computer platform that will finish final data processing. On the other hand, the computer platform sends control instructions to relay control board through the instructions analysis of the main control board so as to control external systems.

Serial communication is used between the main control board and the monitoring computer. The system also provides network communication conditions for further optimization. The system under water adopts distributed structure based on CAN bus, which distributes parts of tasks of central processing node to the corresponding nodes mounted on CAN bus network. Each node can complete its respective function independently and in the meantime it communicates with the main control board. It eases the burden of system's central processing node and ensures the reliability of data transmission. When additional boards are needed, we just simply make them access to CAN bus for system extension [5] [6]. The isolated CAN transceiver which is carried on each board provides differential transceiver capability for CAN controller as well as prevents external noise from flowing into the sensitive circuit of the system.

Based on the flexibility and extendibility of the system, we could add additional boards for system. The category of the extended board depends on application requirements. For instance, in applications of large demand of sensors, we could extend the system by adding acquisition boards. In application of complex external mechanical system, we could extend the system by adding relay control boards.

To implement the function of self-check, power management and closed loop detection structure [7] are applied in order to monitor the state of power supply and system control. 


\section{Hardware design}

We take the design idea of modularization, decomposing the whole into different functional module and integrating corresponding functions on different boards. All boards mount on the CAN bus in the form of nodes and work in collaboration with each other under the management of the main control board. The hardware design follows the principle of generality that the same MCU and isolated CAN transceiver are applied in all boards.

\section{A. Main control management}

As the core management unit of the system, the main control board isn't directly involved in external control or data acquisition except gathering attitude information. It largely reduces the load of main control module.

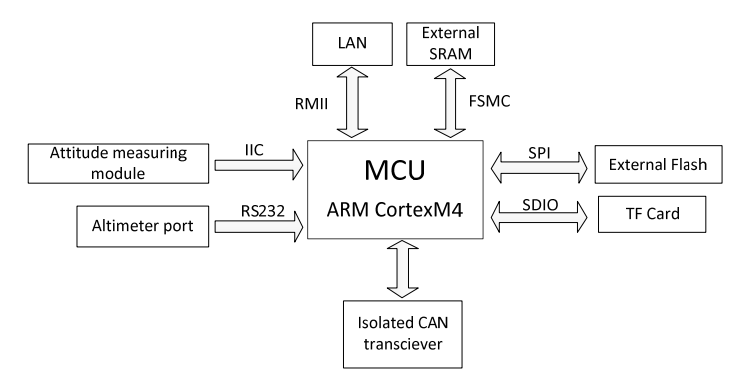

Figure.2. Main control system diagram

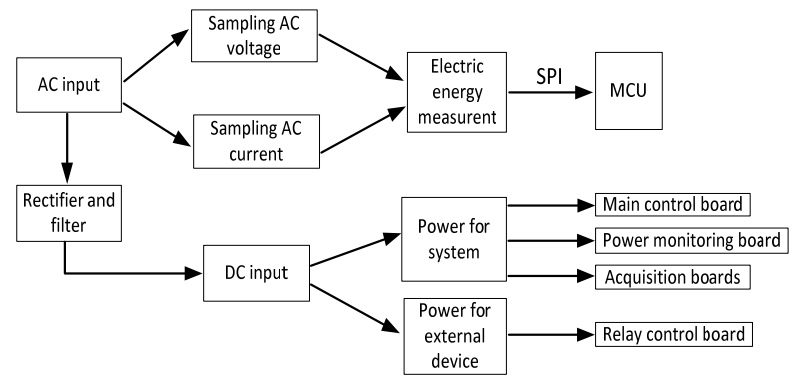

Figure.3. Power management diagram

Figure.2 shows the holistic framework of the main control board. The system's MCU STM32F407ZET6 is based on ARM Cortex-M4 core with frequency up to $168 \mathrm{MHz}$ and memory protection unit. Based on the powerful MCU, rich peripheral resources are configured including network interface, Ethernet transceiver, low power SRAM, serial flash memory, TF Card, attitude measurement module. Besides, the main control system provides several power outputs circuits with large load capacity for external camera systems.

\section{B. Power management}

The power management board consists of rectifier and filter board that is applied to the power supply of the whole system and power monitoring board that is applied to AC input power monitoring. The structure of power management shows in figure.3.

The core unit of power monitoring board is RN8209G, which is an electrical energy measurement chip. With its precise analysis capability, the integrated processing unit inside the chip can implement the measurement of energy and power efficiently. What's more, the power monitoring circuit inside it can ensure the normal work of the chip when the power is off. This self-check ability of power established by such structure contributes to the location and processing of system exception for technicians, improving the reliability and security of the system.

C. function of measurement and control

1) Relay control board

It is in charge of external control. As is showed in figure 4, considering the drawbacks of the structure of open-loop, a closed-loop structure is applied both at the control ports and outputs of relays.

In the control ports, we adopt the detection method with photocouplers which prevents accidental damage to MCU from peripheral systems. The information of the detecting results will be fed back to MCU after MCU sends control instructions so that the system can get access to the state of relays. However, this method cannot be used at the output due to the large output current. Consequently, we apply ACS712 that is a fully integrated and Hall effect-based linear current sensor for measuring the output current of relays so as to obtain the control system's running state. 


\section{2) Acquisition boards}

The acquisition boards whose functions are acquiring data from underwater sensors consist of analog acquisition board and digital acquisition board. Multi-channel partial pressure circuits (including isolated and non-isolated circuits) are designed in this module. It's only necessary for us to modify the values of resistances in acquisition circuits to adapt demands of different sensors owing to its high generality. With isolated operational amplifier of low power consumption, the interference signal will be shielded as well as the system's reliability and security will be improved.

Likewise, taking into account the circuit compatibility, digital acquisition circuits are designed by photocouplers working with three-state buffers inside MCU, which reduce input interference and ensure system's security [8].

\section{System extension}

The extension of the system is quite convenient by utilizing the system's characteristic of plug and play. Extended boards add to CAN bus network equipping with same isolated CAN transceivers as well as follow the same communication protocol. We do not need to make any modification for the original hardware structure of the system.
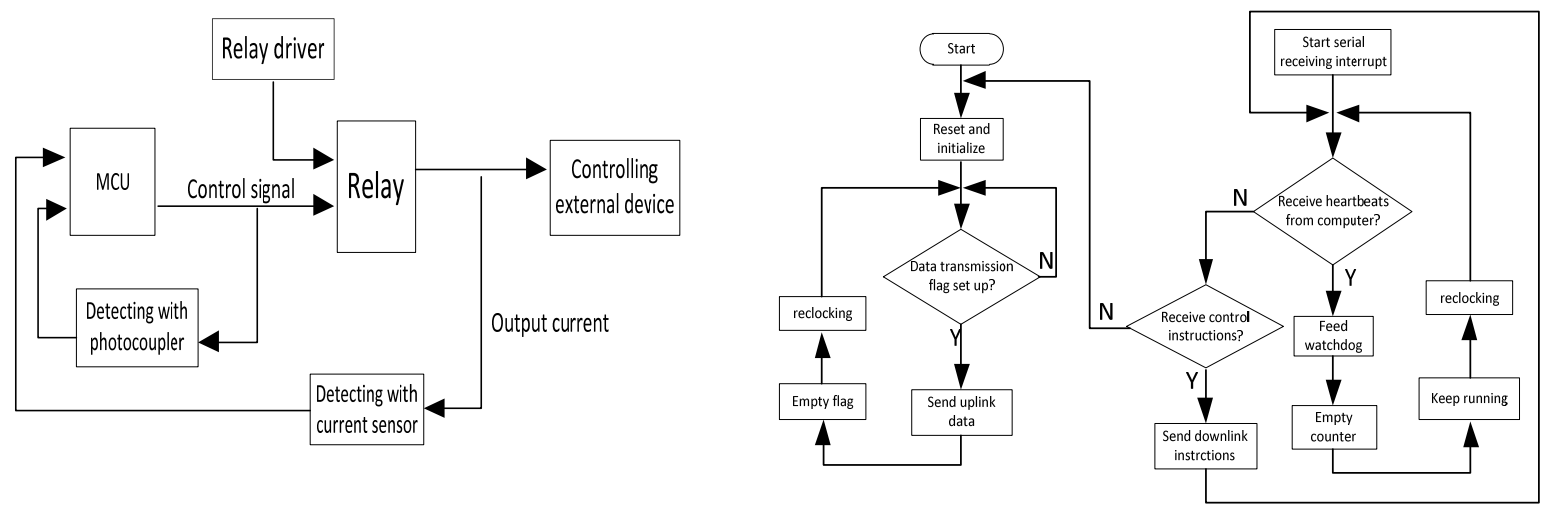

Figure.4. Relay control diagram Figure.5. Software flow chart of main control system

\section{Software design}

The main control board is the core node of the underwater communication network, which manages the operation of the underwater system. Its working process that is given in Figure 5 is composed of the uplink communication and downlink communication. The data transmission flag is set up in the timing control unit of data transmission. The timing control unit that is included in the timer interrupt function sets up the sequential of CAN refreshing and data uploading.

To guarantee the stability and security of the software system, IWDG(Independent Watchdog) is applied. If the main control system receives heartbeat signals from upper computer, it will feed the watchdog, preventing the system from resetting. Otherwise, the system will reset.

We allocate an independent ID for each board mounted on the CAN bus. This ID adopts standard format (11bits) and represents the identifier of data arbitration domain on CAN bus. We define the main control board's ID as 0x000 and the power monitoring board 0x001. The relay control boards' ID start from 0x100 and the acquisition boards' start from 0x200. In this basic system, the relay control board's ID is set to 0x100 and the acquisition boards' ID is set to 0x200 and $0 \times 201$. If the system needs extension, we could set extended boards' ID according to the category they belong to. We just make a slight modification of lower system software and enlarge 
the function of upper system to extend the system conveniently.

To ensure the stability of CAN, we adopt counting unit to monitor CAN communication. The main control system sends refresh frame to CAN bus every $100 \mathrm{~ms}$, updating the control state of relays and calculating the response time of controlled system. The system will re-timing if it receives feedback signals from CAN bus. Otherwise, the system judges the CAN unit break down and it will reset and initialize again. The software flow chart of CAN is given in figure.6.

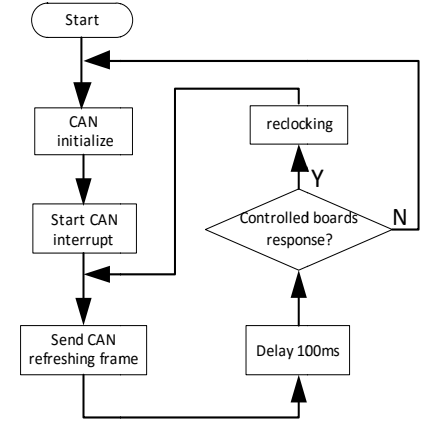

Figure.6. Software flow chart of CAN

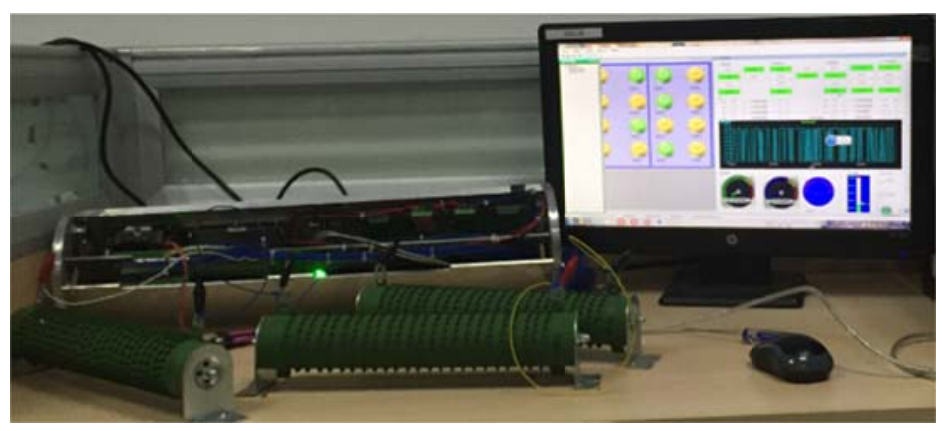

Figure.7. Test environment in laboratory

\section{Test results}

Figure. 7 shows the test environment of this measurement and control system in laboratory. A. Communication and software security test

After powering on, LEDs indicated all boards work properly and the communication was normal. After working for a period of time, we disconnected the main communication. The system reset automatically 5 minutes later which proved the effectiveness of system's security mechanism.

B. Functions tests

1) Function test of self-check

We recorded the values of system's electric energy parameters when we added power resistances as loads. As is shown in figure. 8 and figure.9, input current and apparent power changed along with the change of load. It proved that the system could implement the real-time monitoring.

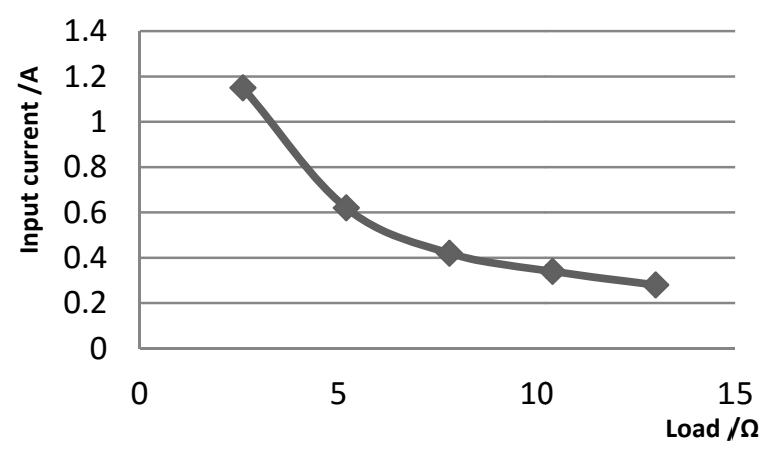

Figure.8. Input current curve

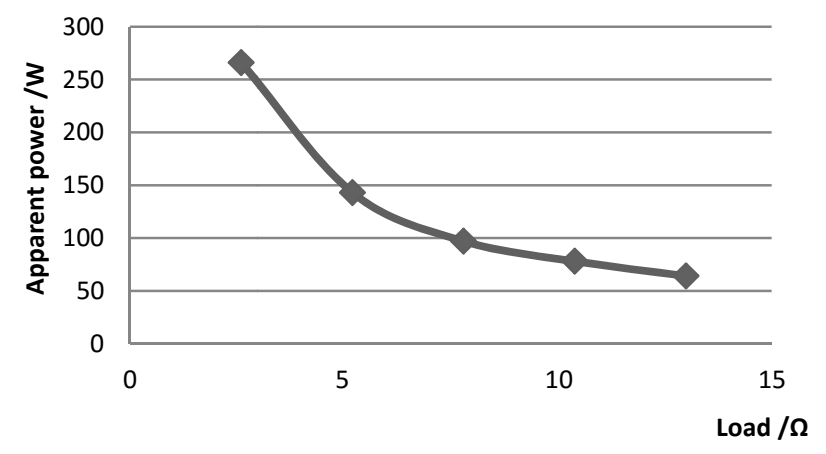

Figure.9. Input apparent power curve

To verify self-check ability on control circuit, we did the opening and closing tests on relays without loads. The corresponding relay buttons could be highlighted when the monitoring computer detected the state feedback of relays. When added with loads, the corresponding relay's output current was displayed on the monitoring computer.

2) Function test of data acquisition

On the one hand, we used precision source measure unit to output voltage signals and current signals so as to simulate analog signals of sensors. The voltage range was from $0 \mathrm{~V}$ to $5 \mathrm{~V}$ with the 
interval of $0.5 \mathrm{~V}$ and the current range was from $4 \mathrm{~mA}$ to $20 \mathrm{~mA}$ with the interval of $1 \mathrm{~mA}$. On the other hand, we used function signal generator to output square waves so as to simulate digital pulse signals of sensors. The frequency range is from $20 \mathrm{~Hz}$ to $2000 \mathrm{~Hz}$.

Tests results shows that input signals can be collected correctly and the system could achieve acquisition functions.

3) Function test of system's extendibility

In order to verify the extendibility of the system, we add a relay control board and an acquisition board for the system, assigning their ID as 0x101 and 0x202. LEDs on these two boards indicate that the system's communication runs normally. Relays on the new added relay control board could be controlled by the monitoring computer and data that new added acquisition board collected could be displayed.

\section{Conclusion}

In this paper, a design of underwater measurement and control system based on distributed management is presented and its main control management, power monitoring, function of measuring and controlling, means of communication and extendibility of system are introduced. Tests results indicate that the system can realize all designed functions. Besides, the system has certain advantages in the aspects of modularization and integration, management efficiency, extendibility, self-check capability and so on, which provide an efficient, stable and reliable measurement and control platform for the operation of new underwater sampling equipment.

\section{Acknowledgement}

This work was partially supported by National High-tech R\&D Program of China (863 Progra m) (Grant No. 2011AA090401) .

\section{Reference}

[1] Deshun Liu, Yongping Jin, Buyan Wan and so on. Review and Development Trends of Deep-sea Mineral Resource Core Sampling Technology and Equipment[J]. China Mechanical engineering. 2014(23).

[2] Xueqiao Geng, Xing Xu, Fanglan Liu and so on. The Current status and Development Trends of Marine Sampling Equipment [J]. Equipment for Geotechnical Engineering . 2009,10(4):11-16.

[3] Qiang Hou, Zhibo Qian. Study of the Distributed Measurement and Control System Based on Network[D]. Northwestern Polytechnical University, 2005.

[4] Gao X, Huang D, Chen Y, et al. The design of a distributed control system based on CAN bus[C]. IEEE International Conference on Mechatronics and Automation. 2013:1118-1122.

[5] Rui Zhao, Kun Yu, Wencheng Zheng and so on. Design and Simulation of the Distributed Motion Control System for Unmanned Underwater Vehicles[J]. Chinese Journal of Ship Research. 2014(6): 92-99.

[6] Qingshu Yang. Research and Realization of the AUV Distributed Control System based on CAN bus[D]. Ocean University of China, 2012.

[7] Yanjun Liu, Jingbiao Liu, Wenyu Cai. Deep-sea Drilling Closed-loop Detection System Based on STM32[J]. Application of Electronic Technique. 2013, 39(6): 17-20. 
[8] Shicheng LÜ, Xiaobo Song, Jing Wang. Design of High-precision Data Acquisition Card Based on LAN[J]. Harbin University of Science and Technology. 2012, 17(2): 22-26. 